\title{
THE FIRST RESULTS FROM STUDIES OF GAMMA DECAY OF PROTON-INDUCED EXCITATIONS AT THE CCB FACILITY*
}

\author{
B. Wasilewska ${ }^{\mathrm{a}}$, M. KMieciK ${ }^{\mathrm{a}}, \mathrm{A} \cdot \mathrm{MAJ}^{\mathrm{a}}$, J. LUKASIK $^{\mathrm{a}}$

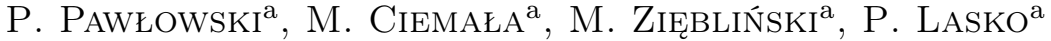 \\ J. Grębosz ${ }^{a}$, F.C.L. Crespi ${ }^{b, c}$, A. BracCo ${ }^{b, c}$, S. Brambilla $^{c}$

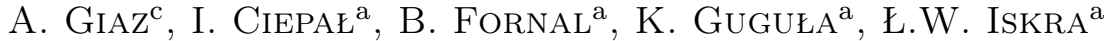 \\ M. KRzysieK ${ }^{\mathrm{a}}$, M. MATEjSKA-Minda ${ }^{\mathrm{d}}$, K. MAZUREK ${ }^{\mathrm{a}}$

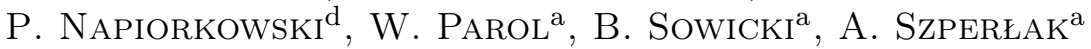 \\ A. TAMII \\ ${ }^{a}$ Institute of Nuclear Physics, PAN, 31-342 Kraków, Poland \\ ${ }^{b}$ Dipartimento di Fisica, University di Milano, 20133 Milano, Italy \\ ${ }^{\mathrm{c}}$ INFN - Milano, 20133 Milano, Italy \\ ${ }^{\mathrm{d}}$ SLCJ, University of Warsaw, Poland \\ ${ }^{\mathrm{e}} \mathrm{RCNP}$, Osaka University, Osaka, Japan
}

(Received December 14, 2016)

A new accelerator facility - Cyclotron Centre Bronowice (CCB) was opened two years ago in Kraków, Poland. Aside from cancer therapy, a scientific program of the nuclear structure research making use of proton beams in the energy range of $70-230 \mathrm{MeV}$ plays a significant role as well. Lead and graphite targets were used to prove the feasibility of exclusive experiments on collective modes in various nuclei. The experimental technique consists of simultaneous energy measurement of scattered beam particles in coincidence with $\gamma$ rays emitted from excited nuclei. This article describes the set-up and the method of analysis used for the experiment.

DOI:10.5506/APhysPolB.48.415

\section{Introduction}

Giant resonances are high-energy excitations of the nucleus, interpreted as collective motions of nucleons forming it. One of them - the Giant Quadrupole Resonance (GQR) - is of particular interest as, after more than 40 years since discovery, only few studies have been made of $\gamma$ decay of such excitation $[1,2]$. In the past, $\gamma$ decay of resonances proved to be

\footnotetext{
* Presented at the Zakopane Conference on Nuclear Physics "Extremes of the Nuclear
} Landscape", Zakopane, Poland, August 28-September 4, 2016. 
an excellent probe to look into deformations of nuclei [3-5], giving a very needed insight into the nuclear Equation of State [6, 7]. The CCB facility provides an opportunity to carry out new studies of GQR $\gamma$ decay.

\section{The experimental set-up}

The experimental technique requires simultaneous measurement of the energy of scattered beam particles and $\gamma$-ray decay from the excited target. As the excitation of different resonances depends on scattering angle, good angular resolution of proton detectors is needed. These requirements are met by using two detector arrays: High Energy $\gamma$-ray deteCTOR (HECTOR [8]) and KRAkow Triple Telescope Array (KRATTA [9]) with the support of plastic scintillators for triggering purposes (Fig. 1).

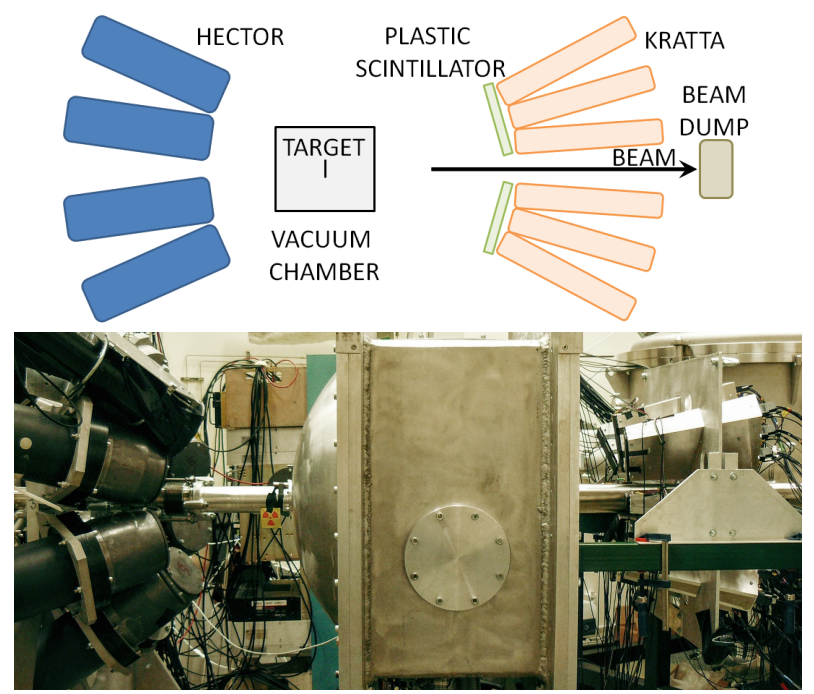

Fig. 1. A schematic view and a photo of the experimental set-up.

The HECTOR array, which is used to measure $\gamma$ rays, consists of 8 large volume $\mathrm{BaF}_{2}$ crystals, characterised by excellent time resolution and efficiency for high-energy $\gamma$-ray detection.

The KRATTA array is a system dedicated to the measurement of energy, emission angle and isotopic composition (via $\Delta E-E$ technique) of light charged particles. A single module consists of two silicon photodiodes (PD), followed by two $\mathrm{CsI}(\mathrm{Tl})$ crystals backed at the end by another PD. For protons, which are the main interest for detection in the described experiments, it is possible to measure energies from 20 to $260 \mathrm{MeV}$. The long rise time of the pulses does not favour, however, using these detectors for triggering purposes. For this reason, thin fast plastic scintillators were placed at the front of them. 
A graphite target was installed in a vacuum chamber, with HECTOR placed at the backward angle of $142^{\circ}$ at a distance of $35 \mathrm{~cm}$ from it, while KRATTA was positioned at forward angles, at a distance of $90 \mathrm{~cm}$. Groups of three KRATTA detectors at the same azimuthal angle were coupled to one common plastic scintillator. The geometry allowed 6 different polar angles to be covered: from 4.3 to $15.3^{\circ}$ with a resolution of $1.8^{\circ}$.

Data from HECTOR and plastics were processed with analogue BaFPro [10] module, while the pulses from KRATTA detectors were digitised using V1732 CAEN boards. Both lines were later combined with the use of Multi Branch System (MBS). The near-line analysis was performed with GREWARE [11] software.

\section{Results}

The data analysis required setting gates on time spectra for all detectors, and gates for protons detected by KRATTA. The energy calibration of KRATTA was performed using elastic scattering reaction at various beam energies, recalculated later to the excitation energy of the target. The energy calibration of HECTOR was done with the use of ${ }^{60} \mathrm{Co}$ and ${ }^{244} \mathrm{Cm}^{-13} \mathrm{C}$ radioactive sources. Two matrices of $\gamma$-ray energy versus excitation energy were created: gated on the HECTOR-plastic coincidence time peak and outside it for the background subtraction.

The background subtracted matrix of $\gamma$-ray energy versus excitation energy is shown in Fig. 2. By setting various conditions on $E^{\star}$ and $E_{\gamma}$, it is possible to study $\gamma$ decays from excited states to low-lying levels including the ground state (Fig. 3). As cross sections for excitation of different modes are dependent on scattering angle [12], choosing only KRATTA detectors placed at the same angle will enable precise selection of the excitation mode to be studied.

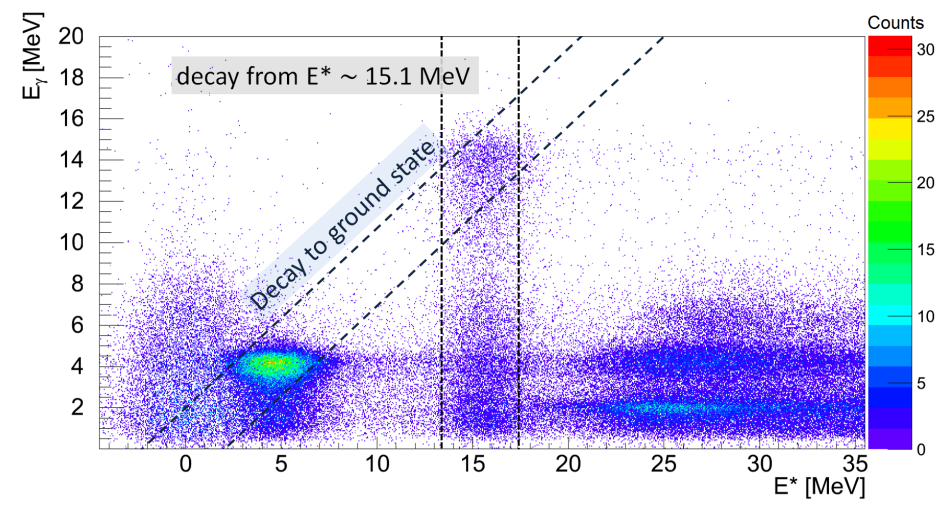

Fig. 2. An $E_{\gamma}$ versus $E^{\star}$ matrix from a measurement with graphite target at proton beam energy of $230 \mathrm{MeV}$. Excitations of the 4.4 and $15.1 \mathrm{MeV}$ levels in ${ }^{12} \mathrm{C}$, with de-excitation via $\gamma$ rays to the ground state, are clearly visible. 

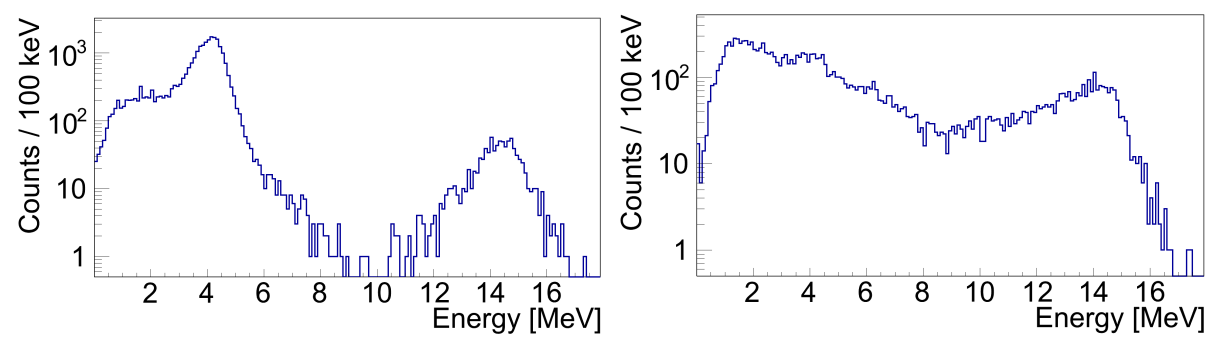

Fig. 3. Examples of spectra produced, by choosing particular gates, from data shown in Fig. 2: (a) $\gamma$ decay of different excited states to the ground state, (b) $\gamma$ decay from $15.1 \mathrm{MeV} 1^{+}$state in ${ }^{12} \mathrm{C}$.

In summary, the presented work demonstrated that the HECTOR + KRATTA set-up at the CCB facility can be used for studies requiring incoincidence measurement of scattered protons and $\gamma$ rays emitted from an excited target. In the near future, experiments aiming to study $\gamma$ decay of GQR in ${ }^{208} \mathrm{~Pb}$ and other nuclei will be performed.

The study is supported by the Polish National Science Centre (NCN) grants Nos.: 2015/17/N/ST2/04034 and 2015/17/B/ST2/01534; and ENSAR2 EU project.

\section{REFERENCES}

[1] J.R. Beene et al., Phys. Rev. C 39, 1307 (1989).

[2] J.R. Beene et al., Phys. Rev. C 41, 920 (1990).

[3] M. Kmiecik et al., Nucl. Phys. A 674, 29 (2000).

[4] A. Maj et al., Nucl. Phys. A 731, 319 (2004).

[5] M. Ciemała et al., Phys. Rev. C 91, 054313 (2015).

[6] P.F. Bortignon, A. Bracco, R.A. Broglia Giant Resonances: Nuclear Structure at Finite Temperature, Harwood Academic, 1998.

[7] M.N. Harakeh, A. van der Woude, Giant Resonances: Fundamental High-Frequency Modes of Nuclear Excitation, Oxford University Press, 2001.

[8] A. Maj et al., Nucl. Phys. A 571, 185 (1994).

[9] J. Eukasik et al., Nucl. Instrum. Methods Phys. Res. A 709, 120 (2013).

[10] C. Boiano et al., in: 2010 IEEE NSS and MIC Conference Record, pp. 268-270.

[11] J. Grębosz, Comput. Phys. Commun. 176, 251 (2007).

[12] A. Schevchenko et al., Phys. Rev. Lett. 93, 122501 (2004). 